\title{
Mathematical models in Physics
}

UDC 533.9

DOI: $10.22363 / 2658-4670-2019-27-4-355-364$

\section{The features of the characteristic electromagnetic plasma bunches oscillations in the long magnetic mirror}

\author{
Andrey A. Novitskiy, Denis V. Chuprov, \\ Vladislav A. Kuznetsov, Eugeniy A. Shevtsov \\ Institute of Physical Research and Technologies \\ Peoples' Friendship University of Russia (RUDN university) \\ 6, Miklukho-Maklaya St., Moscow 117198, Russian Federation
}

(received: December 20, 2019; accepted: December 30, 2019)

The aim of this work was to study the spectrum of LF and HF oscillations generated by plasma bunches created and confined in the volume of a microwave cavity immersed in the magnetic field of a mirror trap. The registration of electrostatic oscillations in the plasma was carried out using two flat electrodes mounted diametrically opposite in the central part of the cavity close to its wall. This diagnostic showed the presence of low-frequency oscillations with frequencies of $130 \mathrm{kHz}$ and $450 \mathrm{kHz}$. The oscillation spectrum in the microwave range was recorded at the minimum of the magnetic trap using a real-time spectrometer and a loosely coupled loop antenna. The registration of the spectra in the $40 \mathrm{MHz}$ band revealed a regular change in the frequency of the fundamental oscillation mode of the cavity and the presence of two harmonics of the synchrotron radiation of the plasma bunch at frequencies of $2.25 \mathrm{GHz}$ and $4.52 \mathrm{GHz}$, respectively. According to the obtained data, the parameters of the formed bunch (density, shape, volume, energy spectra of plasma components) can be restored.

Key words and phrases: gyromagnetic autoresonance, plasma bunches, long magnetic mirror trap, electrostatic and electromagnetic oscillations, spectral analysis

\section{Introduction}

The possibility of generating long-lived plasma bunches with an energetic electronic component under conditions of gyromagnetic autoresonance (GA) in the magnetic field of mirror trap in the regime of a reverse magnetic field has been shown previously [1], [2]. In this mode the reverse pulsed magnetic field is created by coils located at the maxima of the electric component of the microwave field of a standing wave and reduced static magnetic strength to a value that corresponds to the classical electron cyclotron resonance (ECR). Then, the current in the pulse coils decreases, resulting in restoration of the initial profile of the magnetic field produced by the stationary magnetic

(C) Novitskiy A. A., Chuprov D. V., Kuznetsov V.A., Shevtsov E. A., 2019

This work is licensed under a Creative Commons Attribution 4.0 International License http://creativecommons.org/licenses/by/4.0/ 
coils, and the electrons of the produced ECR plasma become trapped in the autoresonant acceleration mode.

The aim of this work was to study the spectrum of LF and HF oscillations generated by plasma bunches created and confined in the mirror trap within microwave cavity.

\section{Experimental setup}

The experimental setup is a cylindrical microwave cavity placed in an axisymmetric magnetic field of a long mirror trap configuration $(R=1.2$, $L=80 \mathrm{~cm}$ ) with magnetic field induction at a trap minimum of $1200 \mathrm{G}$. A stationary magnetic field is created by three pairs of coaxial coils fed by three DC sources.

The pulsed magnetic field required for the implementation of the GA regime is generated by the pulsed current flowing through a pair of coils axisymmetric with the cavity and the coils of the stationary magnetic field. The coils of the pulsed magnetic field are placed symmetrically with respect to the position of the minimum of the magnetic field in the trap in antinodes of the electric field of a standing wave $\mathrm{TE}_{118}$-mode. The $3 \mathrm{D}$-design of the experimental setup, together with the axial distribution of the induction of the resulting magnetic field at different times of the current rise time in the reversing coils of the pulsed field, are presented in Figure 1(a).

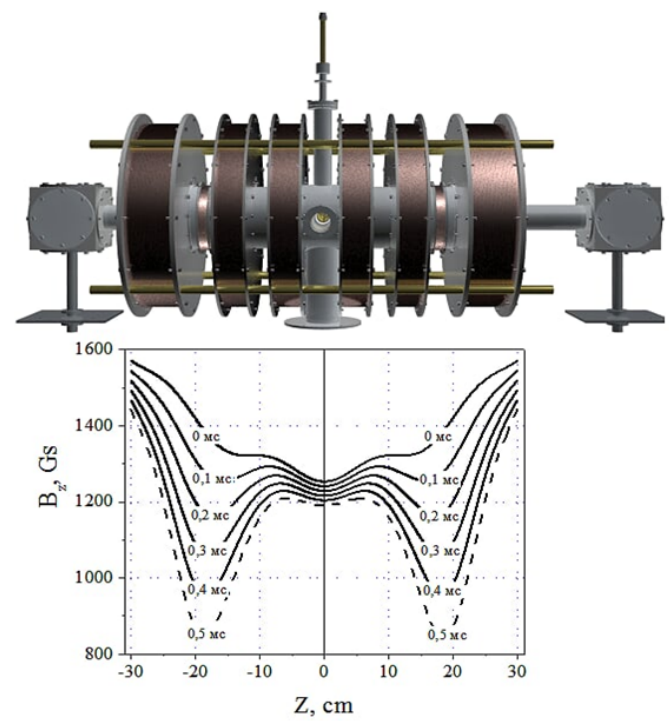

(a) Magnetic system and topology of the magnetic field of the long mirror trap

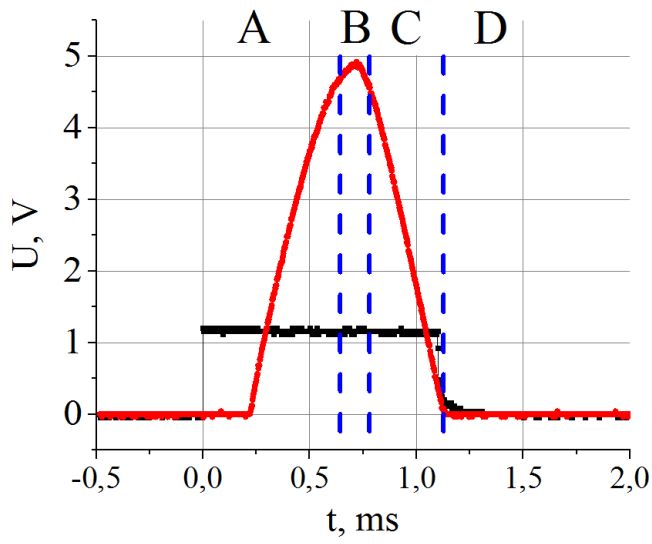

(b) Waveforms describing the characteristic phases of the installation

Figure 1. Microwave cavity

The direction of the current in the pulsed coils provides the creation of a pulsed magnetic field with direction of the induction opposite to the induction of the stationary field, thus reducing locally the resulting magnetic field to a level corresponding to the ECR value for the operating frequency. 
The reduction of the current in the pulse coils and the restoration of the initial profile of the stationary magnetic field in the presence of the microwave field ensure the trapping and acceleration of the initial plasma electrons into the GA regime and the generation of plasma bunches in two symmetrical zones of the setup. The operating cycle of the setup is a current pulse in pulsed magnetic coils with a duration of $900 \mu \mathrm{s}$, synchronized with the forward front of the microwave pulse with a duration of $1.1 \mathrm{~ms}$. The duty cycle of the setup is shown in Figure 1(b): phase A - reduction of the resulting magnetic field to values of the ECR within the two zones of the trap, the phase $\mathrm{B}$ - formation of initial plasma under ECR in two local zones of the trap, phase $\mathrm{C}$ - mode of GA-effect, stage D - confinement mode of the produced plasma bunch in a stationary field of the mirror trap. The restoration of the initial profile of a stationary magnetic field with small gradients leads to the movement of produced bunches to the region of the minimum of the trap and their accumulation. The unit operates in a pulse-periodic mode with a variable duty of GA-cycle. More detailed information about experimental setup and diagnostic methods can be founded from [3], [4].

Registration of electrostatic oscillations in the plasma was carried out using two flat electrodes flush-mounted with the side wall of the cavity at the midplane. The electrodes are made of the metal disks with a diameter of $12 \mathrm{~mm}$ and fixed in the camera ports located in the midplane of the trap, and were oriented parallel to the cavity walls.

The motion of the bunch within the cavity leads to a change in time of the charge induced on the electrostatic probe. The charge distribution in the bunch and its motion dynamics determine the time dependence of the charge induced on the probe. Therefore, by the current taken from the probe through a load resistor of $500 \mathrm{Ohms}$, one can conclude about the bunch parameters and dynamics.

The oscillation spectrum in the microwave range was obtained at the minimum of the magnetic trap using a loop microwave probe. The time and parametric dependences of the frequencies and amplitudes of the recorded oscillations on various discharge conditions were recorded. Microwave signal processing was performed using a Tektronix RSA-6114A spectrum analyzer.

\section{Experimental results}

Processing the voltage waveforms on the load resistance of the wall electrodes using the fast Fourier transform method showed the presence of low-frequency oscillations with frequencies of $130 \mathrm{kHz}$ and $450 \mathrm{kHz}$ at a pressure of $P=1 \cdot 10^{-5}$ Torr, which are observed in the final stage of autoresonance acceleration (delay of $400 \mu \mathrm{s}$ ) (Figure 2).

The motion of an azimuthal and radially asymmetric plasma bunch in the cavity is accompanied by a complex redistribution of its space charge along all three coordinates. An electrostatic probe registers the integrated picture of the distribution of charge density in the near-probe region. The detected oscillations can be due to both the azimuthal and radial inhomogeneity of the bunch, and its axial displacements [5], [6].

Oscillations in the microwave range were recorded synchronously with the signal from the electrostatic probe. The loop microwave probe was introduced into the resonator port, which is at the minimum of the electric 
component of the working mode, which is a transverse circular waveguide located perpendicular to the cavity axis at an angle of $45^{\circ}$ to the oscillation plane of the high-frequency field vector $\mathbf{E}$.

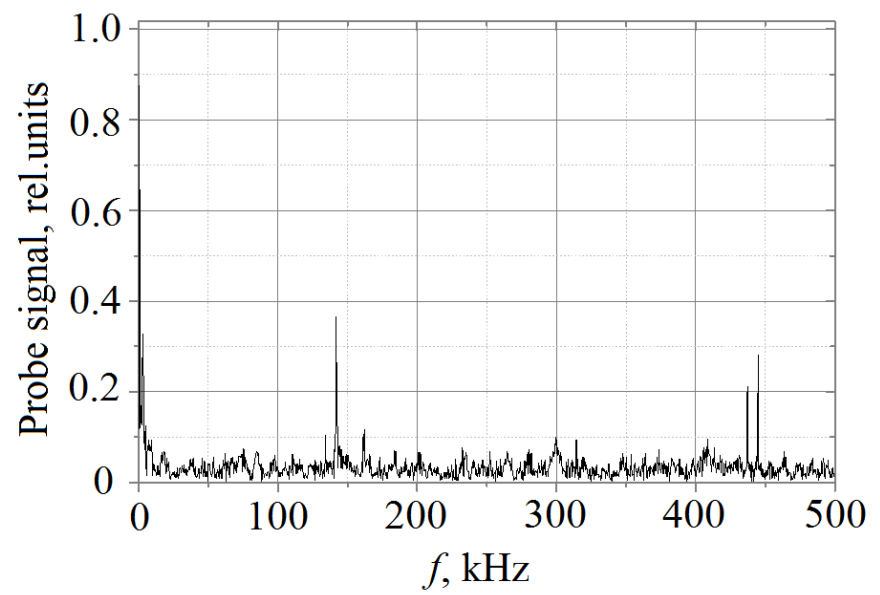

Figure 2. Spectrum of low-frequency oscillations recorded by electrostatic probe placed near the side wall of the cavity in the median plane of the trap

A series of experiments was carried out to obtain high-frequency spectra in the range of the fundamental frequency of oscillations. The measurement results are shown in Figure 3.

A typical spectrogram of high-frequency oscillations at the fundamental frequency of the cavity (Figure 3(a)) illustrates the relatively small magnetron generation bandwidth of the order of $1.5 \mathrm{MHz}$. The central frequency $-f_{H F}$ of the recorded spectral range does not remain constant over time. Figure $3(\mathrm{~b})$ shows the dependence of the frequency corresponding to the distribution maximum on the delay $\Delta t$. GA process starts when the pulsed magnetic field achieves its maximum value. The delay $\Delta t$ is the time interval between the moment of maximum value of pulsed magnetic field and the start trigger of spectrum analyzer. The central frequency of the recorded spectral range decreases quasilinearly with increasing delay. It should be noted that this decrease cannot be due to the delay of the magnetron generation frequency when the complex conductivity of the plasma-loaded resonator changes, since the high-frequency channel uses a standard circuit with an insulator based on a circulator with a matched load that absorbs the reflected power.

Special attention is required to the spectra obtained under the same conditions near the end of the microwave pump pulse. Figure 3(c) shows the spectrogram obtained with a delay $\Delta t=500 \mu \mathrm{s}$ - this moment corresponds to the simultaneous end of microwave and magnetic field pulses (the end of phase $\mathrm{C}$ in Figure 1(b)). It can be seen that in addition to the magnetron generation line, the spectrum contains many other components whose level is 10 or more $\mathrm{dB}$ lower than the signal at the fundamental frequency. This, apparently, is due to the interaction of the standing wave field with bunch particles, which by this time have almost completely formed and begin to shift to the midplane of the mirror trap. 


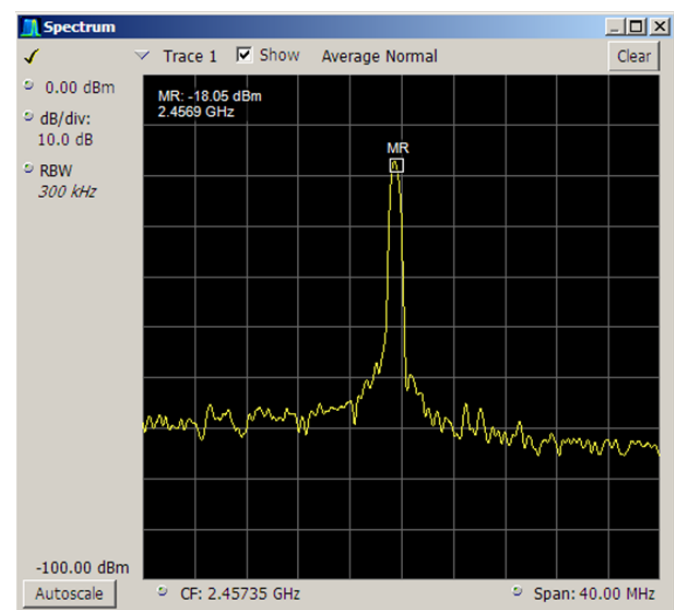

(a) Spectrogram of high-frequency oscillations at the fundamental frequency of the cavity

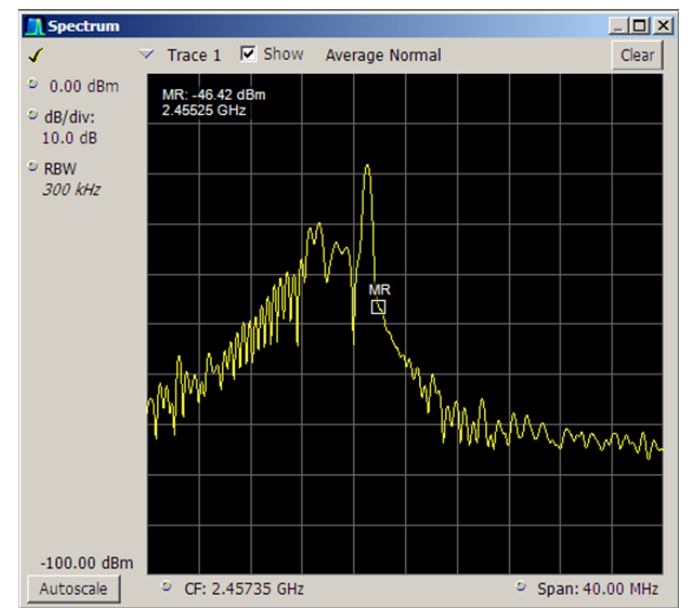

(c) Spectrogram of high-frequency oscillations at the fundamental frequency at $\Delta t=500 \mu \mathrm{s}$

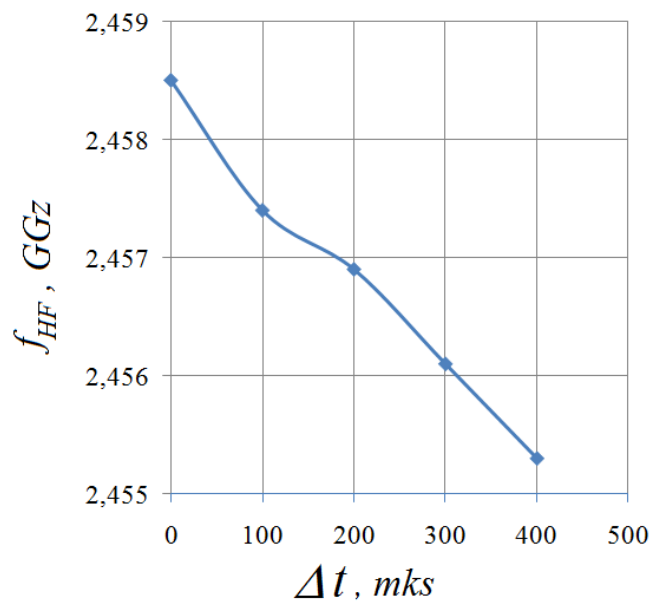

(b) Dependence of the central distribution frequency $f_{H F}$ on the duration $\Delta t$ of the registration delay relative to the maximum of the pulsed magnetic field

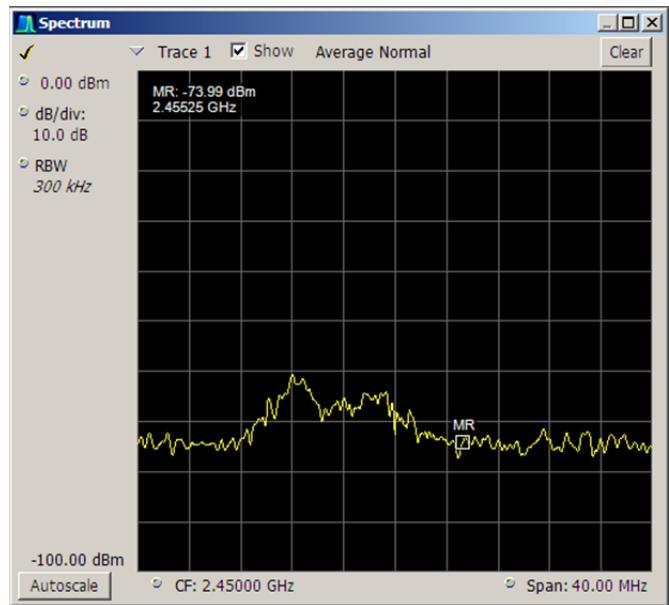

(d) Spectrogram of high-frequency oscillations at $\Delta t=510 \mu \mathrm{s}$

Figure 3. Typical spectrograms of high-frequency oscillations and dependence of the central distribution frequency $f_{H F}$ on the duration $\Delta t$ of the registration delay relative to the maximum of the pulsed magnetic field

Figure 3(d) shows the spectrogram of the signal from the cavity at the moment of $\Delta t=510 \mu \mathrm{s}$. In other words, it is $10 \mu \mathrm{s}$ after the end of the pulse of the magnetron generator. It can be seen that the generation of electromagnetic oscillations at frequencies close to the magnetron frequency continues with a finite attenuation rate. 
Of particular interest are electromagnetic waves due to the collective motion of the particles of the bunch. In fact, this is synchrotron radiation of the bunch electrons accelerated by autoresonance. However, in contrast to classical synchrotrons and vigglers, in which electrons move along strictly defined trajectories, in the discussed plasma storage synchrotron, the bunch particles participate in complex motion along highly non-trivial trajectories. This, of course, affects the spectral composition of the synchrotron radiation of the bunches and its radiation pattern. In the present work, synchrotron radiation was recorded using the same loop microwave probe, which was described above and was used to measure the spectra of microwave oscillations of the cavity at the pump frequency. In Figure 4 the corresponding experimental results are presented.

The upper curve in Figure 4(a) is the integral of the signal recorded from the loop microwave probe in the vicinity of $2.25 \mathrm{GHz}$ in the $40 \mathrm{MHz}$ band by the Tektronix RSA-6114A real-time spectrum analyzer. The lower curve corresponds to the spectral distribution of synchrotron radiation energy in this frequency range. The recorded spectrum has a very complex shape in which several peaks of different widths and degrees of overlap can be distinguished. As a result of this, it would not be right to talk about harmonics of synchrotron radiation, as is done in its classical consideration [7], [8], however, for brevity and definiteness of the present work, we will call radiation recorded in this spectral region the "first harmonic" of synchrotron radiation. It can be assumed that individual peaks in the recorded spectrum correspond to several spatially separated parts of plasma bunches coming from their generation regions near the pulsed-field coils to the confinement region at the midplane of the trap. Interpretation of these results requires additional studies and comparison of their results with the results of measurements of bremsstrahlung and recombination radiation of bunches, as well as data of corpuscular probes in both the radial and axial directions of observation.

The power integral and spectral distribution shown in Figure 4(b) correspond to the "second harmonic" of the synchrotron radiation of a plasma bunch. A spectrogram with a central frequency of $4.52 \mathrm{GHz}$ in the $40 \mathrm{MHz}$ band was obtained with the parameters of the setup and spectrum acquisition unchanged. Nonlinear changes in the spectral composition of synchrotron radiation at the "second harmonic" should be noted.

A series of experiments was carried out with a variation in the working gas pressure, which showed a broadening of the spectrum with increasing pressure.

\section{Conclusion}

Processing waveforms from electrodes using the fast Fourier transform method showed the presence of low-frequency oscillations with frequencies of $130 \mathrm{kHz}$ and $450 \mathrm{kHz}$ at a pressure of $P=1 \cdot 10^{-5}$ Torr, which are observed in the final stage of autoresonant acceleration. The low-frequency components in the emission spectrum of a relativistic bunch arise as a result of complex nonlinear interaction of the bunch particles with external and internal fields, similar to how it happens in a beam discharge [6], in betatrons [7] and in plasma engines [9]. 


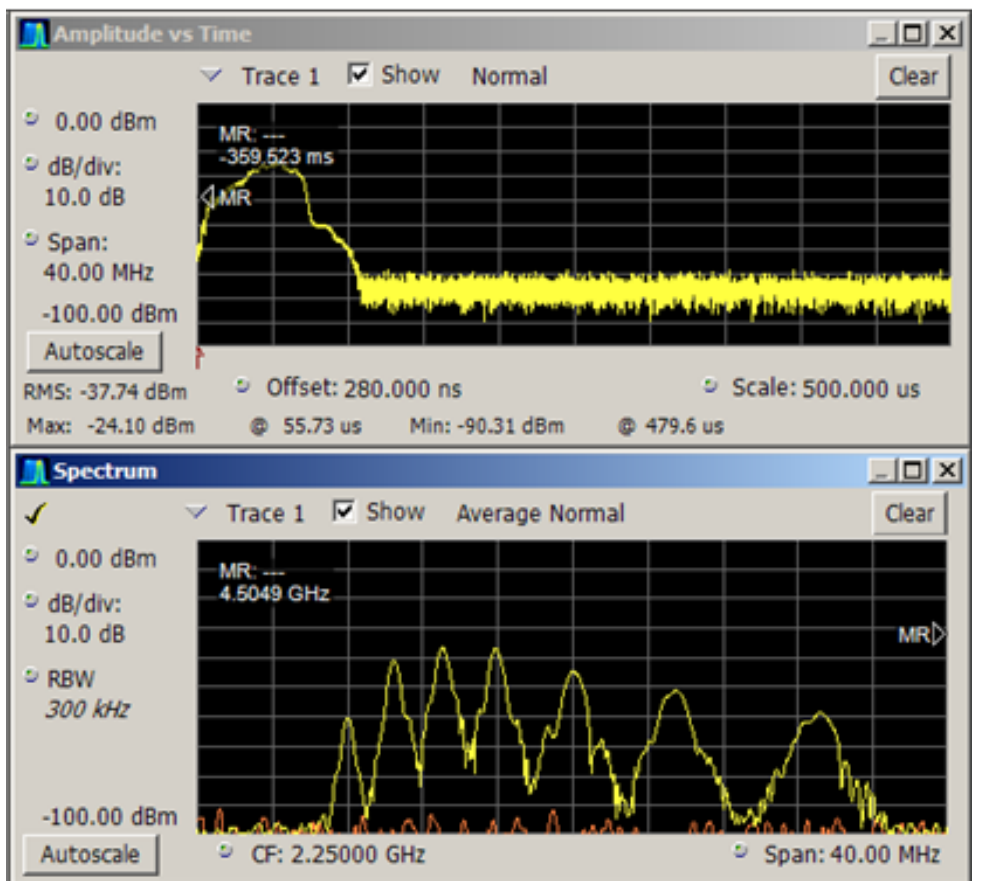

(a) The "first harmonic" $2.25 \mathrm{GHz}$

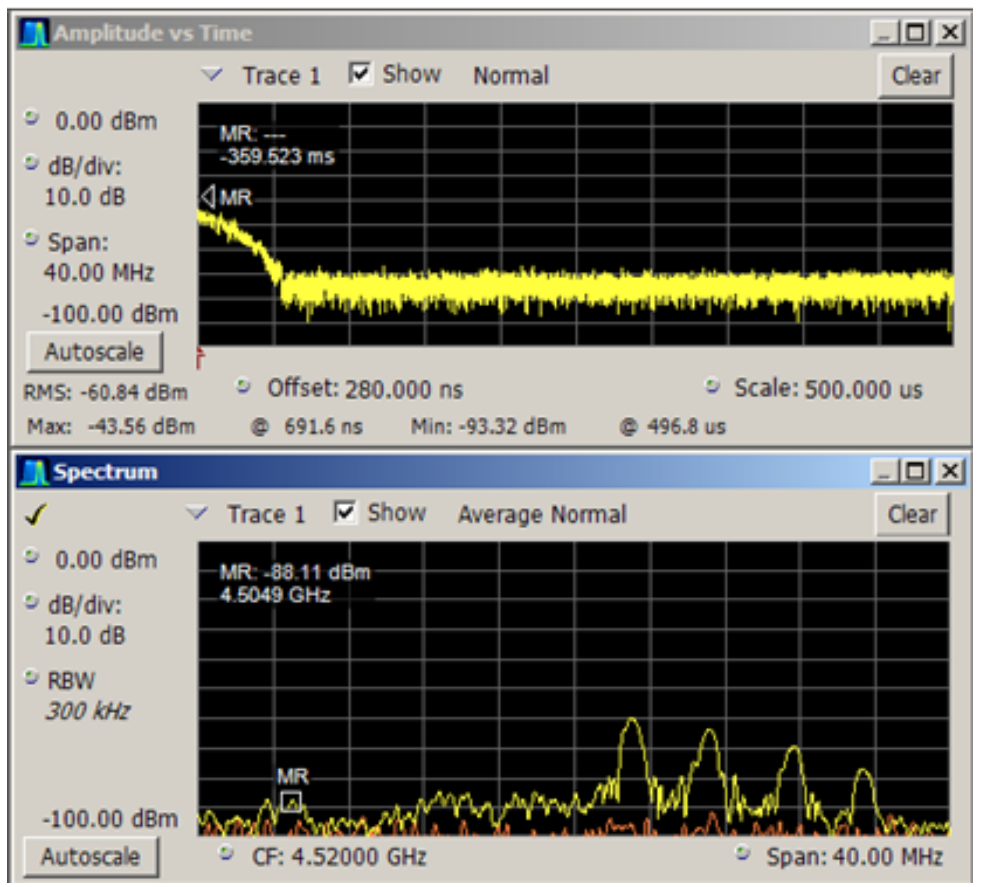

(b) The "second harmonic" $4.52 \mathrm{GHz}$

Figure 4. Spectra of synchrotron radiation 
A comparison of the low-frequency oscillations with the fluctuations in the intensity of the bremsstrahlung detected in the transverse direction, as well as the signals of the PMTs recorded in the region of the trap minimum, shows that they are interconnected and are observed synchronously when the bunches are shifted to the detection region. Microwave signals (at $2.25 \mathrm{GHz}$ and $4.52 \mathrm{GHz}$ ) are also obtained at a given point in time, and a broadening of the spectrum is observed with increasing working gas pressure. Radiation at a frequency of $4.52 \mathrm{GHz}$ was obtained in a trap at the end of a microwave pump pulse.

\section{Acknowledgments}

The authors appreciate the support of the "RUDN University Program $5-100$ ", which allowed to prepare the section 2 of the paper. For the results described in the sections 3 and 4, some of us (AAN, DVC) also appreciate the financial support from the Ministry of Education and Science of the Russian Federation (agreement 3.2223.2017.4.6).

\section{References}

[1] V. V. Andreev, A. A. Novitsky, and A. M. Umnov, "Spectral changes of bremsstrahlung plasma bunch generated under autoresonance in a long mirror," IOP Conf. Series: Journal of Physics: Conf. Series, vol. 1094, no. 9, p. 012 013, 2018. DOI: 10.1088/1742-6596/1094/1/012013.

[2] V. V. Andreev, A. A. Novitsky, and D. V. Chuprov, "The Use of Streak Photography, X-Ray Radiography, and Radiometric and Spectrometric Measurements to Study Plasma Bunches Generated under Gyroresonant Interactions," Physics of Atomic Nuclei, vol. 82, no. 10, pp. 1-10, 2019. DOI: $10.1134 / \mathrm{S} 1063778819100016$.

[3] V. V. Andreev and A. M. Umnov, "Relativistic plasma and electron bunches in plasma synchrotrons of GYRAC," Plasma Sources Science and Technology, vol. 8, no. 3, pp. 479-487, Jan. 1999. DOI: 10.1088/09630252/8/3/318.

[4] V. V. Andreev, D. V. Chuprov, V. I. Ilgisonis, A. A. Novitsky, and A. M. Umnov, "Gyromagnetic autoresonance plasma bunches in a magnetic mirror," Physics of Plasmas, vol. 24, no. 9, p. 093 518, 2017. DOI: 10. 1063/1.4986009.

[5] R. W. Gould, "Dynamics of Non-Neutral Plasmas," Physics of plasmas, vol. 2, no. 6, pp. 2151-2163, 1995. DOI: 10.1063/1.871302.

[6] S. M. Krivoruchko and E. A. Kornilov, "Excitation of Low-frequency Oscillations and Control of Two-stream Instability Spectra," JETP Letters, vol. 10, no. 10, pp. 299-301, 1969.

[7] T. J. Smith, J. Golden, and C. A. Kapetanakos, "Studies of synchrotron radiation emission from the modified betatron accelerator," Journal of Applied Physics, vol. 69, no. 10, pp. 6836-6843, 1991. DOI: $10.1063 / 1$. 347673. 
[8] R. C. Davidson and S. M. Mahajan, "Synchrotron Radiation Spectrum for an Intense Relativistic E-Layer," Physics of Fluids, vol. 17, pp. 22672274, 1974. DOI: 10.1063/1.1694702.

[9] K. P. Kirdyashev, A. I. Bugrova, A. V. Desyatkov, and V. K. Kharchevnikov, "Low-frequency spectra of electron oscillations in an SPD-ATON stationary plasma thruster," Technical Physics Letters, vol. 35, no. 2, pp. 158-161, 2009. DOI: 10.1134/S1063785009020187.

\section{For citation:}

A. A. Novitskiy, D. V. Chuprov, V. A. Kuznetsov, E. A. Shevtsov, The features of the characteristic electromagnetic plasma bunches oscillations in the long magnetic mirror, Discrete and Continuous Models and Applied Computational Science 27 (4) (2019) 355-364. DOI: 10.22363/2658-4670-2019-27-4-355-364.

\section{Information about the authors:}

Andrey A. Novitskiy (Russian Federation) - Head of Laboratory of the Institute of Physical Research and Technologies, Peoples' Friendship University of Russia (RUDN University) (e-mail: novitskiy-aa@rudn.ru, phone: $+7(915) 4013703$, ORCID: https://orcid.org/0000-0002-0248-1640, ResearcherID: O-8773-2017, Scopus Author ID: 56763990400)

Denis V. Chuprov (Russian Federation) - Senior Lecturer of the Institute of Physical Research and Technologies, Peoples' Friendship University of Russia (RUDN University) (e-mail: chuprov-dv@rudn.ru, phone: $+7(926) 1342628$, ORCID: https://orcid.org/0000-0002-6768-6196, ResearcherID: O-3193-2013, Scopus Author ID: 6508067157)

Vladislav A. Kuznetsov (Russian Federation) - Student of the Institute of Physical Research and Technologies, Peoples' Friendship University of Russia (RUDN University) (e-mail: 1032162058@rudn.ru, phone: +7(985)8318472, ORCID: https://orcid.org/0000-0002-3707-157X)

Eugeniy A. Shevtsov (Russian Federation) - Student of the Institute of Physical Research and Technologies, Peoples' Friendship University of Russia (RUDN University) (e-mail: 1032162067@rudn.ru, phone: +7(968)9201311, ORCID: https://orcid.org/0000-0001-7685-3630) 
УДК 533.9

DOI: $10.22363 / 2658-4670-2019-27-4-355-364$

\title{
Особенности характерных электромагнитных колебаний плазменных сгустков в длинном пробкотроне
}

\author{
А. А. Новицкий, Д. В. Чупров, В. А. Кузнецов, Е. А. Шевцов \\ Институт физических исследований и технологий \\ Российский университет дружбы народов \\ ул. Миклухо-Маклая, д. 6, Москва, 117198, Россия
}

Целью данной работы являлось изучение спектра НЧ и ВЧ колебаний генерируемых плазменными сгустками, создаваемыми и удерживаемыми в рабочем объеме высокочастотного резонатора, находящегося в магнитном поле зеркальной ловушки. Регистрация электростатических колебаний в плазме осуществлялось при помощи двух плоских электродов установленных диаметрально противоположно в центральной части резонатора в его пристеночной области. Эта диагностика показала наличие низкочастотных колебаний с частотами 130 кГц и 450 кГц. Спектр колебаний в СВЧ диапазоне регистрировался в минимуме магнитной ловушки при помощи спектрометра реально времени и слабо связанной петлевой антенны. Регистрация спектров в полосе 40 МГц позволила выявить закономерное изменение частоты основной моды колебаний резонатора и наличие двух гармоник синхротронного излучения плазменного сгустка на частотах 2.25 ГГц и 4.52 ГГц соответственно. По полученным данным могут быть восстановлены параметры сформированного сгустка (плотность, форма, объем, энергетические спектры компонент плазмы).

Ключевые слова: гиромагнитный авторезонанс, плазменные сгустки, протяженный пробкотрон, электростатические и электромагнитные колебания, спектральный анализ 